\title{
EDITORIAL \\ New frontiers in phenotypic plasticity and evolution
}

\author{
Heredity (2015) 115, 273-275; doi:10.1038/hdy.2015.64
}

The past decade has seen a surge of interest in phenotypic plasticity. From the thousands of citations of West-Eberhard's volume (West-Eberhard, 2003), to the emphasis on plasticity in the 'Extended Evolutionary Synthesis' presented recently by Laland et al. (2014) in Nature, evolutionary biologists are increasingly considering the possibility that plasticity can influence the direction of evolution. Plasticity has long been considered a ubiquitous feature of organisms, allowing them to respond to environmental variation, often in an adaptive manner (for example, Baldwin, 1896; Clausen et al., 1940; Levins, 1968). In particular, plasticity is thought to have a major role in evolution by allowing survival in novel environments ('Baldwin Effect' sensu Simpson (1953)), and facilitating the accumulation and release of cryptic genetic variation (Suzuki and Nijhout, 2006; Crispo et al., 2010; Hoffmann and Sgró, 2011). More recently, the importance of plasticity has been stressed in the evolution of complex and integrated phenotypes (Bateson and Gluckman, 2011; Robinson and Beckerman, 2013).

Although much recent research on the role of plasticity emphasizes the positive effects that plasticity may have on evolution and diversification (Price et al., 2003; Pfennig et al., 2010; Moczek et al., 2011), it is also true that plasticity can buffer the impact of selection as it can result in the production of adaptive phenotypes without genetic change in populations experiencing novel environments (Huey et al., 2003). These alternative possibilities mean that research on the role of plasticity in evolution is particularly relevant today given its potential importance in the face of rapid human-induced environmental change (for example, Sih, 2013). Will more plastic species be more likely to survive in novel and rapidly changing environments, forming the basis of future diversification events?

This special issue highlights some of the many exciting and open research questions concerning phenotypic plasticity: is plasticity costly, how does it affect diversification, does developmental timing matter? This thematic issue stems from some of the topics covered at the 2012 symposium 'Phenotypic Plasticity, Alteration, Variation and Speciation', at Lund University, Sweden. These papers touch on the controversial issue of whether and how adaptive plasticity can accelerate evolution; methodological issues concerning appropriate analyses of phenotypic plasticity in many-dimensional shape space; and the interplay of developmental timing, behaviour and flexible, and integrated phenotypes. This special issue gathers papers by leading scientists conducting research on the evolution of phenotypic plasticity that examine the importance of plasticity in development and evolution. These papers include literature reviews, theoretical research and empirical research to present a comprehensive thematic issue that should be of broad interest to evolutionary biologists, developmental biologists and ecologists alike.

Here we highlight four general themes across this special issue.

\section{PLASTICITY AND THE DEVELOPMENT OF INTEGRATED, COMPLEX PHENOTYPES}

Plasticity is an integral part of organismal development that allows for robustness in the face of nearly ubiquitous environmental perturbations. This view of plasticity has evolved from the relatively simple and static view of one trait at one developmental time point, to wholeorganism and whole-life cycle perspectives. Several papers in this special issue reflect this view by highlighting the importance of plasticity in the development of integrated, complex phenotypes.

Forsman (this issue) advocates a research approach that takes the whole organism into consideration, arguing that trait integration needs more attention and that instead of focusing on single traits we need to view the complexity of the complete phenotype. Such a perspective should be extended, not only to the individual, but also to the level of populations and species and to performance and ecological success.

Moczek (this issue) discuss the utility of two conceptual frameworks, facilitated variation and niche construction, highlighting how these concepts can link studies of developmental plasticity to the evolution of complex phenotypes. This review also contrasts gene and genome-centric perspectives in development and explores several major contributions made by combining developmental plasticity with evolutionary biology (see other themes below).

Nilsson-Örtman et al. focuses on plasticity across ontogeny. Their study highlights how damselfly larvae experience temperature variation across a latitudinal gradient and present complex patterns over ontogeny in thermal reaction norms. They describe their corresponding genetic variance in growth rate across populations and species, with the results suggesting that genetic variance of the height and slope of thermal reaction norms increase with age.

\section{PLASTICITY AND INDIVIDUAL VARIATION}

The importance of plasticity in development means that individuals in a population often adopt completely different morphological and behavioural traits as a result of experiences during development. Such individual variation has important implications for both the ecology and evolution of populations (for example, Bolnick et al., 2003). Bateson (this issue) focuses on this question of why individuals are so different from each other. His review reflects on the many different processes of phenotypic plasticity, and discusses how temporal dimensions, organisational levels, nutritional factors at an early developmental stage and selection or developmental instructions generate differences among individuals. In order to completely understand the evolution of phenotypic plasticity, a multidimensional view is essential in future research.

Collyer et al. take a methodological approach to the question of individual variation. There are unique challenges associated with quantifying individual variation, especially when the number of phenotypic variables exceeds the number of individuals under 
consideration. Collyer et al. present a method for handling many-dimensional data describing multidimensional phenotypes. By implementing landmark-based geometric morphometric data, the authors provide a non-parametric method of evaluating effect size for such data sets, allowing more morphological information to be processed without compromising statistical power.

\section{COSTS AND LIMITS IN THE EVOLUTION OF PLASTICITY}

This issue also touches on a classic topic in the study of plasticity-costs and limits. Murren et al. reflect on the decades of literature offering mixed support for costs of plasticity; why are costs absent or difficult to detect when theory and intuition suggests they should be ubiquitous? The authors suggest that the type of plasticity matters, but also argue that many situations can obscure or ameliorate costs, such as if costs are measured later in life, or if individuals vary in quality or access to resources.

A research review by Baker et al. discusses female life history plasticity in threespine sticklebacks (Gasterosteus aculeatus) and focuses on four life-history traits that are directly involved with reproductive output, reviewing observed plasticity in each of these traits and exploring the potential limits to plasticity in them. They also attempt to assess the costs and benefits of plasticity in each trait separately and in total. Finally, they differentiate between traits in which plastic expression may be rapid vs those for which the plasticity is only observed across reproductive seasons. Unusually, the authors have comparable data both from laboratory and field studies, which provides rarely available insights.

Although costs are frequently addressed in the plasticity literature, evolutionary constraints and limits are less frequently addressed. Snell-Rood et al. use a comparative approach to test the idea that the evolution of phenotypic plasticity is constrained by the relative time in an organism's ontogeny that environmental information is received. If organisms obtain cues at an early stage of their ontogeny, which initiate and provide more opportunity for developmental plasticity, this should incur greater variation in the final trait. This has been described as the epiphenotype hypothesis and Snell-Rood and colleagues test these assumptions in heterochronic shifts by contrasting precocial and altricial birds.

Bourdeau et al. focus on variation in selection for plasticity. They use a meta-analysis approach to show that marine and freshwater snails are shaped by different selective forces and for that reason show significant variation for the magnitude of phenotypic plasticity.

\section{PLASTICITY AND DIVERSIFICATION}

Over the last decade, there has been increasing interest in the role of plasticity in diversification, and in the conditions under which plasticity may promote or retard evolutionary change. Foster et al. revisited adaptive radiation in a model system, the threespine stickleback (Gasterosteus aculeatus). Phenotypic plasticity can influence phenotypic integration, and if new phenotypes are exposed along with cryptic genetic variation, it may result in novel trajectories of evolutionary change. The authors discuss why a comprehensive understanding of adaptive radiations in sticklebacks can increase our understanding how trait correlations have evolved.

Aubret asks whether plasticity can accelerate evolutionary change through 'the Baldwin expediting effect', using multiple tiger snake populations across southern Australia. The results suggest that at an early stage of island colonisation, directional selection, combined with adaptive plasticity, have enhanced the rate of evolution towards the optimal local phenotype, providing strong empirical support for the Baldwin expediting effect.

Arendt (this issue) explores the role of dispersal plasticity in diversification and speciation. Plasticity in the timing of dispersal, or habitat choice in dispersal, can have major consequences for speciation, in some cases accelerating it, or in other cases breaking down reproductive barriers. This review discusses the available theory and data on this open question.

In summary, this issue highlights how the study of phenotypic plasticity has not only progressed over the last few decades, but is also still addressing open and exciting questions. In particular, plasticity is recognised as an important feature of organismal development that results in the expression of complex, integrated and robust phenotypes in the face of environmental variation. The costs and limits associated with plasticity must be considered within the context of the organism's ontogeny, life history and evolutionary history. Plasticity has important consequences for the expression of individual variation, along with diversification and speciation. Plasticity has emerged as an important concept that links developmental and organismal perspectives to ecological and evolutionary questions. Future decades are bound to see plasticity as a common bridge linking different subdisciplines of biology, from genomics and development to ecology and evolution.

\section{DATA ARCHIVING}

There were no data to deposit.

\section{CONFLICT OF INTEREST}

The authors declare no conflict of interest.

\section{ACKNOWLEDGEMENTS}

We thank the Swedish Research Council and the Aquatic Ecology Unit, Lund University for financial support of the 2012 symposium 'Phenotypic Plasticity, Alteration, Variation and Speciation', at Lund University, Sweden.

J Hollander ${ }^{1}$, E Snell-Rood ${ }^{2}$ and S Foster ${ }^{3}$ ${ }^{1}$ Aquatic Ecology, Department of Biology, Lund University, Lund, Sweden; ${ }^{2}$ Department of Ecology, Evolution and Behavior, University of Minnesota, Saint Paul, MN, USA and ${ }^{3}$ Department of Biology, Clark

University, Worcester, MA, USA

E-mail: johan.hollander@biol.lu.se

Baldwin JM (1896). A new factor in evolution. Am Nat 30: 441-451.

Bateson P, Gluckman P (2011). Plasticity, Robustness, Development and Evolution. Cambridge University Press: Cambridge, UK.

Bolnick DI, Svanbäck R, Fordyce JA, Yang LH, Davis JM, Hulsey CD et al. (2003). The ecology of individuals: incidence and implications of individual specialization. Am Nat 161: 1-28.

Clausen J, Keck DD, Hiesey WM (1940). Experimental Studies on the Nature of Species. I. Effect of Varied Environments on Western North American Plants. Carnegie Institution of Washington: Washington, DC, USA.

Crispo E, DiBattista JD, Correa C, Thibert-Plante X, McKellar AE, Schwartz AK et al. (2010). The evolution of phenotypic plasticity in response to anthropogenic disturbance. Evol Ecol Res 12: 47-66.

Hoffmann AA, Sgró CM (2011). Climate change and evolutionary adaptation. Nature 470 $479-485$.

Huey RB, Hertz PE, Sinervo B (2003). Behavioral drive versus behavioral inertia in evolution: a null model approach. Am Nat 161: 357-366.

Laland KN, Uller T, Feldman MW, Sterelny K, Muller GB, Moczek et al. (2014). Does evolutionary theory need a rethink? Nature 514: 161-164. 
Levins R (1968). Evolution in Changing Environments. Princeton University Press: Princeton, NJ, USA.

Moczek AP, Sultan S, Foster S, Ledón-Rettig C, Dworkin I, Nijhout HF et al. (2011). The role of developmental plasticity in evolutionary innovation. Proc $R$ Soc Lond $B$ 278: 2705-2713.

Pfennig DW, Wund MA, Snell-Rood EC, Cruickshank T, Schlichting CD, Moczek AP (2010).

Phenotypic plasticity's impacts on diversification and speciation. Trends Ecol Evol 25: 459-467.

Price TD, Qvarnström A, Irwin DE (2003). The role of phenotypic plasticity in driving genetic evolution. Proc $R$ Soc Lond B 270: 1433-1440.
Robinson MR, Beckerman AP (2013). Quantifying multivariate plasticity: genetic variation in resource acquisition drives plasticity in resource allocation to components of life history. Ecol Lett 16: 281-290.

Sih A (2013). Understanding variation in behavioural responses to human-induced rapid environmental change: a conceptual overview. Anim Behav 85: 1077-1088.

Simpson GG (1953). The Baldwin effect. Evolution 7: 110-117.

Suzuki Y, Nijhout HF (2006). Evolution of a polyphenism by genetic accommodation. Science 311: 650-652.

West-Eberhard MJ (2003). Developmental Plasticity and Evolution. Oxford University Press: New York, USA. 